\title{
Measurement of the Electron Antineutrino Oscillation with 1958 Days of Operation at Daya Bay
}

D. Adey, ${ }^{1}$ F. P. An, ${ }^{2}$ A. B. Balantekin, ${ }^{3}$ H. R. Band, ${ }^{4}$ M. Bishai,${ }^{5}$ S. Blyth,${ }^{6,7}$ D. Cao, ${ }^{8}$ G. F. Cao, ${ }^{1}$ J. Cao, ${ }^{1}$ Y. L. Chan, ${ }^{9}$ J. F. Chang, ${ }^{1}$ Y. Chang, ${ }^{7}$ H. S. Chen, ${ }^{1}$ S. M. Chen, ${ }^{10}$ Y. Chen, ${ }^{11}$ Y. X. Chen, ${ }^{12}$ J. Cheng, ${ }^{13}$ Z. K. Cheng, ${ }^{14}$ J. J. Cherwinka, ${ }^{3}$ M. C. Chu, ${ }^{9}$ A. Chukanov, ${ }^{15}$ J. P. Cummings, ${ }^{16}$ F. S. Deng, ${ }^{17}$ Y. Y. Ding, ${ }^{1}$ M. V. Diwan, ${ }^{5}$ M. Dolgareva, ${ }^{15}$ D. A. Dwyer,${ }^{18}$ W. R. Edwards, ${ }^{18}$ M. Gonchar, ${ }^{15}$ G. H. Gong, ${ }^{10}$ H. Gong, ${ }^{10}$ W. Q. Gu, ${ }^{5}$ L. Guo, ${ }^{10}$ X. H. Guo, ${ }^{19}$ Y. H. Guo, ${ }^{20}$ Z. Guo, ${ }^{10}$ R. W. Hackenburg, ${ }^{5}$ S. Hans, ${ }^{5,}{ }^{*}$ M. He,${ }^{1}$ K. M. Heeger, ${ }^{4}$ Y. K. Heng, ${ }^{1}$ A. Higuera, ${ }^{21}$ Y. B. Hsiung, ${ }^{6}$ B. Z. Hu, ${ }^{6}$ J. R. Hu, ${ }^{1}$ T. Hu, ${ }^{1}$ Z. J. Hu, ${ }^{14}$ H. X. Huang, ${ }^{22}$ X. T. Huang, ${ }^{13}$ Y. B. Huang, ${ }^{1}$ P. Huber,${ }^{23}$ W. Huo, ${ }^{17}$ G. Hussain, ${ }^{10}$ D. E. Jaffe, ${ }^{5}$ K. L. Jen, ${ }^{24}$ X. L. Ji, ${ }^{1}$ X. P. Ji, ${ }^{5}$ R. A. Johnson, ${ }^{25}$ D. Jones, ${ }^{26}$ L. Kang, ${ }^{27}$ S. H. Kettell, ${ }^{5}$ L. W. Koerner, ${ }^{21}$ S. Kohn, ${ }^{28}$ M. Kramer, ${ }^{18,28}$ T. J. Langford, ${ }^{4}$ L. Lebanowski, ${ }^{10}$ J. Lee, ${ }^{18}$ J. H. C. Lee, ${ }^{29}$ R. T. Lei, ${ }^{27}$ R. Leitner, ${ }^{30}$ J. K. C. Leung, ${ }^{29}$ C. Li,${ }^{13}$ F. Li, ${ }^{1}$ H. L. Li ${ }^{13}$ Q. J. Li ${ }^{1}$ S. Li, ${ }^{27}$ S. C. Li ${ }^{23}$ S. J. Li,${ }^{14}$ W. D. Li,${ }^{1}$ X. N. Li ${ }^{1}$ X. Q. Li,${ }^{31}$ Y. F. Li, ${ }^{1}$ Z. B. Li, ${ }^{14}$ H. Liang,${ }^{17}$ C. J. Lin, ${ }^{18}$ G. L. Lin,${ }^{24}$ S. Lin, ${ }^{27}$ S. K. Lin, ${ }^{21}$ Y.-C. Lin, ${ }^{6}$ J. J. Ling, ${ }^{14}$ J. M. Link, ${ }^{23}$ L. Littenberg, ${ }^{5}$ B. R. Littlejohn, ${ }^{32}$ J. C. Liu, ${ }^{1}$ J. L. Liu, ${ }^{33}$ Y. Liu, ${ }^{13}$ Y. H. Liu,${ }^{8}$ C. W. Loh,${ }^{8}$ C. Lu, ${ }^{34}$ H. Q. Lu, ${ }^{1}$ J. S. Lu, ${ }^{1}$ K. B. Luk,${ }^{28,18}$ X. B. Ma, ${ }^{12}$ X. Y. Ma, ${ }^{1}$ Y. Q. Ma, ${ }^{1}$ Y. Malyshkin, ${ }^{35}$ C. Marshall, ${ }^{18}$ D. A. Martinez Caicedo,${ }^{32}$ K. T. McDonald, ${ }^{34}$ R. D. McKeown,${ }^{36,37}$ I. Mitchell, ${ }^{21}$ L. Mora Lepin, ${ }^{35}$ J. Napolitano, ${ }^{26}$ D. Naumov, ${ }^{15}$ E. Naumova, ${ }^{15}$ J. P. Ochoa-Ricoux, ${ }^{35}$ A. Olshevskiy, ${ }^{15}$ H.-R. Pan, ${ }^{6}$ J. Park, ${ }^{23}$ S. Patton, ${ }^{18}$ V. Pec, ${ }^{30}$ J. C. Peng, ${ }^{38}$ L. Pinsky, ${ }^{21}$ C. S. J. Pun,${ }^{29}$ F. Z. Qi,${ }^{1}$ M. Qi,${ }^{8}$ X. Qian,,${ }^{5}$ R. M. Qiu, ${ }^{12}$ N. Raper, ${ }^{14}$ J. Ren, ${ }^{22}$ R. Rosero, ${ }^{5}$ B. Roskovec, ${ }^{35}$ X. C. Ruan, ${ }^{22}$ H. Steiner, ${ }^{28,18}$ J. L. Sun, ${ }^{39}$ W. Tang, ${ }^{5}$ D. Taychenachev,${ }^{15}$ K. Treskov, ${ }^{15}$ W.-H. Tse, ${ }^{9}$ C. E. Tull, ${ }^{18}$ B. Viren, ${ }^{5}$ V. Vorobel, ${ }^{30}$ C. H. Wang, ${ }^{7}$ J. Wang, ${ }^{14}$ M. Wang, ${ }^{13}$ N. Y. Wang, ${ }^{19}$ R. G. Wang, ${ }^{1}$ W. Wang, ${ }^{37,14}$ W. Wang, ${ }^{8}$ X. Wang, ${ }^{40}$ Y. F. Wang, ${ }^{1}$ Z. Wang, ${ }^{1}$ Z. Wang, ${ }^{10}$ Z. M. Wang, ${ }^{1}$ H. Y. Wei, ${ }^{5}$ L. H. Wei, ${ }^{1}$ L. J. Wen, ${ }^{1}$ K. Whisnant, ${ }^{41}$ C. G. White, ${ }^{32}$ T. Wise, ${ }^{4}$ H. L. H. Wong, ${ }^{28,18}$ S. C. F. Wong, ${ }^{14}$ E. Worcester, ${ }^{5}$ Q. Wu, ${ }^{13}$ W. J. Wu, ${ }^{1}$ D. M. Xia, ${ }^{42}$ Z. Z. Xing, ${ }_{1}^{1}$ J. L. Xu, ${ }^{1}$ T. Xue, ${ }^{10}$ C. G. Yang, ${ }^{1}$ H. Yang, ${ }^{8}$ L. Yang, ${ }^{27}$ M. S. Yang, ${ }^{1}$ M. T. Yang, ${ }^{13}$ Y. Z. Yang, ${ }^{14}$ M. Ye, ${ }^{1}$ M. Yeh, ${ }^{5}$ B. L. Young, ${ }^{41}$ H. Z. Yu, ${ }^{14}$ Z. Y. Yu, ${ }^{1}$ B. B. Yue, ${ }^{14}$ S. Zeng, ${ }^{1}$ L. Zhan, ${ }^{1}$ C. Zhang, ${ }^{5}$ C. C. Zhang, ${ }^{1}$ F. Y. Zhang, ${ }^{33}$ H. H. Zhang, ${ }^{14}$ J. W. Zhang, ${ }^{1}$ Q. M. Zhang, ${ }^{20}$ R. Zhang, ${ }^{8}$ X. F. Zhang, ${ }^{1}$ X. T. Zhang, ${ }^{1}$ Y. M. Zhang, ${ }^{14}$ Y. M. Zhang, ${ }^{10}$ Y. X. Zhang, ${ }^{39}$ Y. Y. Zhang, ${ }^{33}$ Z. J. Zhang, ${ }^{27}$ Z. P. Zhang, ${ }^{17}$ Z. Y. Zhang, ${ }^{1}$ J. Zhao, ${ }^{1}$ P. Zheng, ${ }^{27}$ L. Zhou, ${ }^{1}$ H. L. Zhuang, ${ }^{1}$ and J. H. Zou ${ }^{1}$

(The Daya Bay Collaboration)

${ }^{1}$ Institute of High Energy Physics, Beijing

${ }^{2}$ Institute of Modern Physics, East China University of Science and Technology, Shanghai

${ }^{3}$ University of Wisconsin, Madison, Wisconsin 53706

${ }^{4}$ Wright Laboratory and Department of Physics, Yale University, New Haven, Connecticut 06520

${ }^{5}$ Brookhaven National Laboratory, Upton, New York 11973

${ }^{6}$ Department of Physics, National Taiwan University, Taipei

${ }^{7}$ National United University, Miao-Li

${ }^{8}$ Nanjing University, Nanjing

${ }^{9}$ Chinese University of Hong Kong, Hong Kong

${ }^{10}$ Department of Engineering Physics, Tsinghua University, Beijing

${ }^{11}$ Shenzhen University, Shenzhen

${ }^{12}$ North China Electric Power University, Beijing

${ }^{13}$ Shandong University, Jinan

${ }^{14}$ Sun Yat-Sen (Zhongshan) University, Guangzhou

${ }^{15}$ Joint Institute for Nuclear Research, Dubna, Moscow Region

${ }^{16}$ Siena College, Loudonville, New York 12211

${ }^{17}$ University of Science and Technology of China, Hefei

${ }^{18}$ Lawrence Berkeley National Laboratory, Berkeley, California 94720

${ }^{19}$ Beijing Normal University, Beijing

${ }^{20}$ Department of Nuclear Science and Technology, School of Energy and Power Engineering, Xi'an Jiaotong University, Xi'an

${ }^{21}$ Department of Physics, University of Houston, Houston, Texas 77204

${ }^{22}$ China Institute of Atomic Energy, Beijing

${ }^{23}$ Center for Neutrino Physics, Virginia Tech, Blacksburg, Virginia 24061

${ }^{24}$ Institute of Physics, National Chiao-Tung University, Hsinchu 


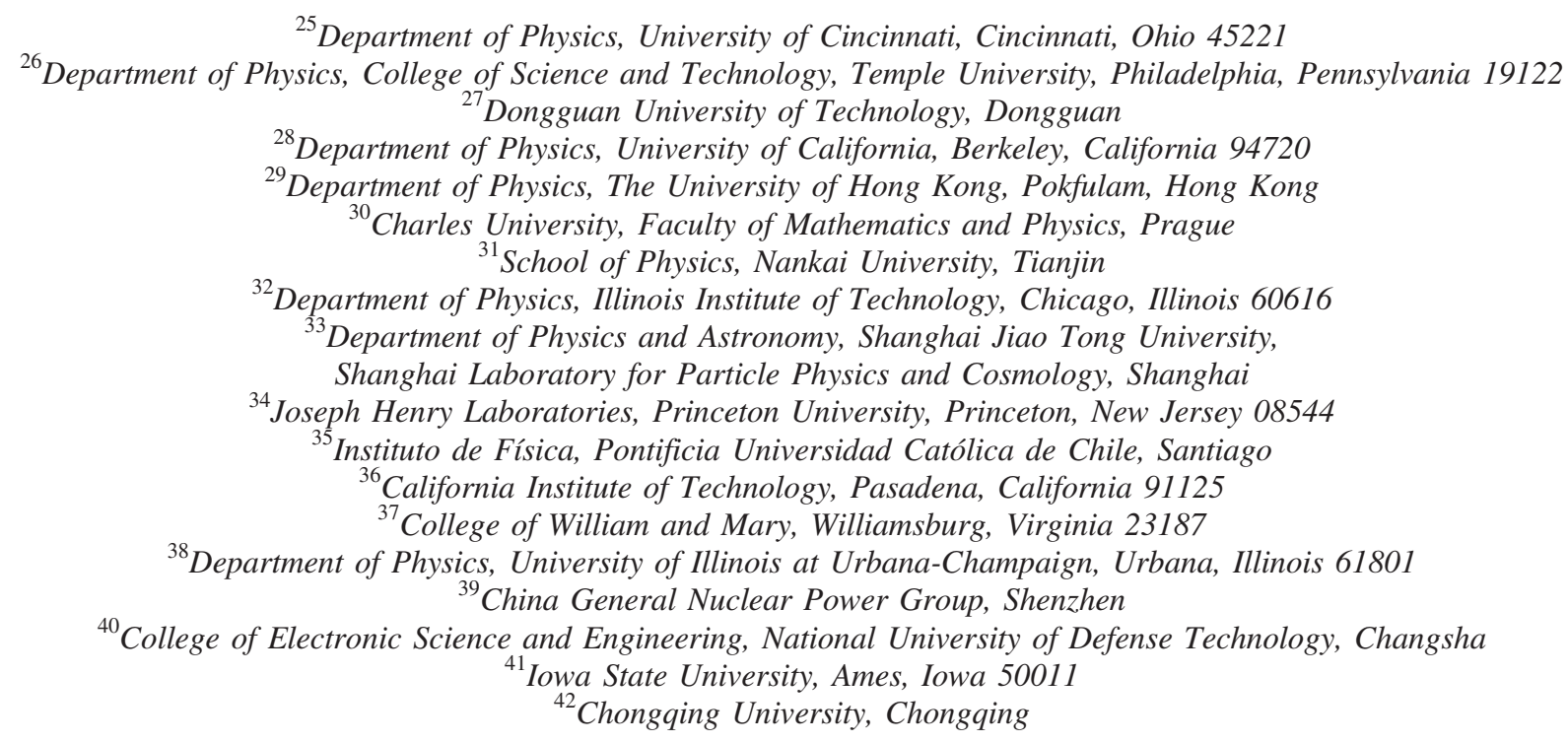

(Received 11 September 2018; published 14 December 2018)

\begin{abstract}
We report a measurement of electron antineutrino oscillation from the Daya Bay Reactor Neutrino Experiment with nearly 4 million reactor $\bar{\nu}_{e}$ inverse $\beta$ decay candidates observed over 1958 days of data collection. The installation of a flash analog-to-digital converter readout system and a special calibration campaign using different source enclosures reduce uncertainties in the absolute energy calibration to less than $0.5 \%$ for visible energies larger than $2 \mathrm{MeV}$. The uncertainty in the cosmogenic ${ }^{9} \mathrm{Li}$ and ${ }^{8} \mathrm{He}$ background is reduced from $45 \%$ to $30 \%$ in the near detectors. A detailed investigation of the spent nuclear fuel history improves its uncertainty from $100 \%$ to $30 \%$. Analysis of the relative $\bar{\nu}_{e}$ rates and energy spectra among detectors yields $\sin ^{2} 2 \theta_{13}=0.0856 \pm 0.0029$ and $\Delta m_{32}^{2}=\left(2.471_{-0.070}^{+0.068}\right) \times 10^{-3} \mathrm{eV}^{2}$ assuming the normal hierarchy, and $\Delta m_{32}^{2}=-\left(2.575_{-0.070}^{+0.068}\right) \times 10^{-3} \mathrm{eV}^{2}$ assuming the inverted hierarchy.
\end{abstract}

DOI: 10.1103/PhysRevLett.121.241805

Neutrino flavor oscillation driven by the $\theta_{13}$ mixing angle has been observed using reactor antineutrinos [1-3] and accelerator neutrinos $[4,5]$. The Daya Bay experiment previously reported the observation of a nonzero value of $\sin ^{2} 2 \theta_{13}$ via the disappearance of reactor antineutrinos over $\sim$ kilometer distances [1], and a measurement of the effective mass-squared difference $\Delta m_{e e}^{2}$ via the distortion of the $\bar{\nu}_{e}$ energy spectrum [6]. Both of these measurements based on 1230 days of operation are described in detail in Ref. [7]. This Letter presents a measurement of these two parameters with a data set acquired in 1958 days of stable operation, and with several improvements to the analysis when compared with previous measurements.

The Daya Bay experiment consists of eight identically designed antineutrino detectors (ADs), two in each near experimental halls (EH1 and $\mathrm{EH} 2$ ), and four in the far hall

Published by the American Physical Society under the terms of the Creative Commons Attribution 4.0 International license. Further distribution of this work must maintain attribution to the author(s) and the published article's title, journal citation, and DOI. Funded by SCOAP.
(EH3). Antineutrinos are produced by six reactor cores, with two cores $\sim 365 \mathrm{~m}$ from EH1 and four cores $\sim 505 \mathrm{~m}$ from $\mathrm{EH} 2$. The average geometric baseline to $\mathrm{EH} 3$ over all six cores is $1663 \mathrm{~m}$. Each AD consists of three nested cylindrical vessels. The inner acrylic vessel is filled with 20-t $0.1 \%$ gadolinium-doped liquid scintillator (Gd LS), which serves as the primary $\bar{\nu}_{e}$ target. The acrylic vessel surrounding the target is filled with undoped LS, increasing the efficiency for detecting $\gamma$ rays produced in the target. A total of 192 8-in. photomultiplier tubes (PMTs) are uniformly positioned on the cylinder of the outermost stainless steel vessel and immersed in mineral oil. The experimental setup is described in detail in Refs. [8,9].

Stable data taking began on 24 December 2011 with six ADs. The final two ADs were installed between 28 July and 19 October 2012 in EH2 and EH3. Operation of the 8-AD configuration continued until 20 December 2016. A special calibration was performed to better understand the neutron detection efficiency [10] and the optical shadowing of the calibration source enclosures. In January 2017, the Gd LS in EH1 AD1 was replaced by purified LS to study the light yields with different recipes and purification methods for next generation experiments. The remaining seven ADs 
resumed data taking on 26 January 2017. Since the statistical precision of the oscillation measurement is driven by the interaction rate in the far detectors, the impact of removing $\mathrm{EH} 1 \mathrm{AD} 1$ is negligible. The results presented in this Letter combine the data from all three periods: $6 \mathrm{AD}$ (217 days), $8 \mathrm{AD}$ (1524 days), and $7 \mathrm{AD}$ (217 days).

Reactor antineutrinos are detected via the inverse $\beta$-decay (IBD) reaction, $\bar{\nu}_{e}+p \rightarrow e^{+}+n$. The positron deposits kinetic energy in the scintillator and annihilates with an electron, forming a prompt signal, which gives an estimate of the $\bar{\nu}_{e}$ energy, $E_{\bar{\nu}_{e}} \approx E_{\text {prompt }}+0.78 \mathrm{MeV}$. Neutron capture on Gd emits several $\gamma$ rays with a mean total energy of $8.05 \mathrm{MeV}$, forming a delayed signal with a mean capture time of $\sim 30 \mu \mathrm{s}$. The coincidence between the prompt and delayed signals efficiently discriminates IBD reactions from background.

The primary goal of energy calibration is to reduce potential energy scale variations among the ADs, which dominate the systematic error in the oscillation measurement. PMT gains are calibrated once per day using thermal noise hits. Light yields $(\sim 160$ photoelectrons/MeV) are found to be decreasing by $\sim 1 \%$ per year, and are calibrated with $\gamma$ rays from ${ }^{60} \mathrm{Co}$ sources deployed weekly. The $15 \%$ spatial nonuniformity, and its $<0.5 \%$ per year time dependence, are corrected with ${ }^{60} \mathrm{Co}$ sources along three calibration axes. Calibration references with different spatial distributions are examined, including $\mathrm{Gd}$ and ${ }^{1} \mathrm{H}$ neutron captures, as well as artificially and naturally occurring $\alpha$ and $\gamma$ particles. The reconstructed energies for these particles are compared among ADs and found to be consistent within $0.2 \%$, which is taken as the energy scale uncertainty uncorrelated among detectors. Consistent results are obtained by an alternative method using spallation neutron capture on Gd to calibrate the energy scale, time dependence, and nonuniformity [7].

The absolute energy response, which relates the actual and observed prompt energies, is improved. The readout system underestimates the charge of the PMT signals when they overlap in time due to the AC coupling of the front end electronics. This results in a nonlinear response of the charge over the entire detector at the $\sim 10 \%$ level in the energy region of interest. Effects of scintillator quenching and Cherenkov radiation contribute an additional $\sim 10 \%$ nonlinearity. The energy nonlinearity model with $1 \%$ precision used in previous results is described in Ref. [7]. The model is constructed with energies of $\gamma$ rays from deployed and natural sources, and the $\beta$ spectrum from cosmogenic ${ }^{12} \mathrm{~B}$. To improve the understanding of both electronics and LS nonlinearity, dedicated calibrations have been performed.

In December 2015, a full flash ADC (FADC) readout system was installed in EH1 AD1, recording PMT waveforms at $1 \mathrm{GHz}$ and 10-bit resolution. The FADC and the existing electronics readout system acquire data simultaneously. A deconvolution method is applied to the waveforms to minimize the dependence on the single photoelectron pulse shape, in particular the overshoot, and to extract the integrated charge with minimum bias [11]. The residual nonlinearity in the reconstructed charge from a single waveform is estimated to be less than $1 \%$ from an electronics simulation tuned to data. An event-byevent comparison of the total charge of the two readout systems gives a measurement of the existing system's nonlinearity at $0.2 \%$ precision.

Uncertainties in the visible energy from $\gamma$ rays (previously $\sim 1 \%$ ) are dominated by the poor knowledge of optical shadowing by source enclosures $(5 \mathrm{~cm}$ tall and $2 \mathrm{~cm}$ in diameter cylinders). A special calibration campaign in January 2017 deployed ${ }^{60}$ Co sources with PTFE, greenish Teflon, and stainless steel enclosures absorbing $<0.10 \%$, $1.22 \%$, and $0.65 \%$ of photons, respectively, as determined from the simulation. The reconstructed energies of data and simulation agree to $0.2 \%$ for all source enclosures. The total uncertainty from these $\gamma$ rays is improved to $0.5 \%$, including the residual nonuniformity between point-like $\gamma$-ray sources, which preferentially illuminate the detector center, and IBD events over the full target volume.

The $\beta$ decay of ${ }^{12} \mathrm{~B}$ is an allowed transition of the Gamow-Teller type with a $Q$ value of $13.4 \mathrm{MeV}$. A total of 470000 cosmogenic ${ }^{12} \mathrm{~B}$ candidates are observed in the Gd LS of the four near ADs. The $\beta$ spectrum of ${ }^{12} \mathrm{~B}$ decay is compared to a prediction that includes Fermi motion, screening effects, corrections for the finite size of the nucleus, and weak magnetism. A significant uncertainty in the prediction is due to the weak magnetism correction, estimated as a linear correction with a coefficient of $(0.48 \pm 0.24) \% \mathrm{MeV}[12,13]$. This uncertainty is propagated to the nonlinearity model together with uncertainties in the decay branching ratios.

The functional form of the nonlinearity model used in this analysis is identical to the one reported in Ref. [7]. The improved prompt energy response is shown in Fig. 1.

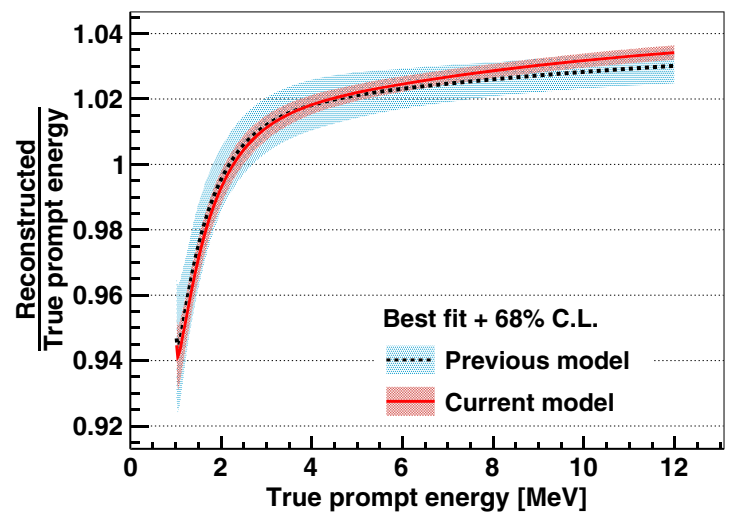

FIG. 1. Relationship between the reconstructed and true prompt energy, which is a combination of positron kinetic energy and energies of the annihilation $\gamma$ rays. The updated model and its uncertainty (red) contains improvements described in the text. The previous model [7] is shown for comparison. 
TABLE I. Summary of signal and backgrounds. Rates are corrected for the muon veto and multiplicity selection efficiencies $\varepsilon_{\mu} \cdot \varepsilon_{m}$. The procedure for estimating accidental, fast neutron, Am-C, and $(\alpha, n)$ backgrounds is unchanged from Ref. [7].

\begin{tabular}{|c|c|c|c|c|c|c|c|c|}
\hline & \multicolumn{2}{|c|}{ EH1 } & \multicolumn{2}{|c|}{$\mathrm{EH} 2$} & \multicolumn{4}{|c|}{ EH3 } \\
\hline & AD1 & AD2 & AD3 & AD8 & AD4 & AD5 & AD6 & AD7 \\
\hline $\bar{\nu}_{e}$ candidates & 830036 & 964381 & 889171 & 784736 & 127107 & 127726 & 126666 & 113922 \\
\hline DAQ live time (days) & 1536.621 & 1737.616 & 1741.235 & 1554.044 & 1739.611 & 1739.611 & 1739.611 & 1551.945 \\
\hline$\varepsilon_{\mu} \times \varepsilon_{m}$ & 0.8050 & 0.8013 & 0.8369 & 0.8360 & 0.9596 & 0.9595 & 0.9592 & 0.9595 \\
\hline Accidentals $\left(\right.$ day $\left.^{-1}\right)$ & $8.27 \pm 0.08$ & $8.12 \pm 0.08$ & $6.00 \pm 0.06$ & $5.86 \pm 0.06$ & $1.06 \pm 0.01$ & $1.00 \pm 0.01$ & $1.03 \pm 0.01$ & $0.86 \pm 0.01$ \\
\hline Fast neutron $\left(\mathrm{AD}^{-1} \mathrm{day}^{-1}\right)$ & \multicolumn{2}{|c|}{$0.79 \pm 0.10$} & \multicolumn{2}{|c|}{$0.57 \pm 0.07$} & \multicolumn{4}{|c|}{$0.05 \pm 0.01$} \\
\hline${ }^{9} \mathrm{Li} /{ }^{8} \mathrm{He}\left(\mathrm{AD}^{-1}\right.$ day $\left.^{-1}\right)$ & \multicolumn{2}{|c|}{$2.38 \pm 0.66$} & \multicolumn{2}{|c|}{$1.59 \pm 0.49$} & \multicolumn{4}{|c|}{$0.19 \pm 0.08$} \\
\hline Am-C correlated $\left(\right.$ day $\left.^{-1}\right)$ & $0.17 \pm 0.07$ & $0.15 \pm 0.07$ & $0.14 \pm 0.06$ & $0.13 \pm 0.06$ & $0.06 \pm 0.03$ & $0.05 \pm 0.02$ & $0.05 \pm 0.02$ & $0.04 \pm 0.02$ \\
\hline${ }^{13} \mathrm{C}(\alpha, n){ }^{16} \mathrm{O}\left(\mathrm{day}^{-1}\right)$ & $0.08 \pm 0.04$ & $0.06 \pm 0.03$ & $0.04 \pm 0.02$ & $0.06 \pm 0.03$ & $0.04 \pm 0.02$ & $0.04 \pm 0.02$ & $0.04 \pm 0.02$ & $0.04 \pm 0.02$ \\
\hline $\bar{\nu}_{e}$ rate $\left(\right.$ day $\left.^{-1}\right)$ & $659.36 \pm 1.00$ & $81.09 \pm 0.9$ & $601.83 \pm 0.82$ & $595.82 \pm 0.85$ & $74.75 \pm 0.23$ & $75.19 \pm 0.23$ & $74.56 \pm 0.23$ & $75.33 \pm 0.24$ \\
\hline
\end{tabular}

The precision is better than $0.5 \%$ for prompt energy larger than $2 \mathrm{MeV}$. In-flight annihilation and the three $\gamma$-rays decay from orthopositronium have a $<0.1 \%$ impact on the energy model. The precision is limited by the systematic uncertainties associated with the $\gamma$-ray samples at prompt energies below $3 \mathrm{MeV}$, and by the statistics of the ${ }^{12} \mathrm{~B}$ sample at higher energy. Consistent results are obtained with the removal of any one $\gamma$ ray, the measured electronics linearity, or the ${ }^{12} \mathrm{~B}$ constraint. A tabulated form of the model is provided in the Supplemental Material [14].

IBD candidates are selected following the same criteria as Selection A in Ref. [7]. The estimated signal and background rates, as well as the efficiencies of the muon veto, $\epsilon_{\mu}$, and multiplicity selection, $\epsilon_{m}$, are summarized in Table I. More than $3.9 \times 10^{6} \bar{\nu}_{e}$ candidates are identified. In all three halls, the background is smaller than $2 \%$, and contributes less than $0.15 \%$ to the uncertainty on the IBD rate. Consistent results are obtained using Selection B in Ref. [7].

The dominant background uncertainty is due to the cosmogenic production of ${ }^{9} \mathrm{Li}$ and ${ }^{8} \mathrm{He}$ (referred as ${ }^{9} \mathrm{Li}$ in the following) with subsequent $\beta-n$ decay, which cannot be distinguished from IBD on an event-by-event basis. Yields are estimated by fitting the distribution of time between the IBD candidate and the preceding muon, as shown in Fig. 2 for muons with visible energy $E_{\mu}^{\text {rec }}$ between 1 and $1.8 \mathrm{GeV}$ in EH1. The falloff with increasing time depends only on the muon rate for muon-uncorrelated events, while muoninduced ${ }^{9} \mathrm{Li}$ decays with a lifetime of $257 \mathrm{~ms}$.

A prompt energy cut is applied to enhance the contribution from ${ }^{9} \mathrm{Li}$, which has a higher energy spectrum. In the previous analysis, the cut was 3.5 to $12 \mathrm{MeV}$, resulting in the distribution shown in the top panel of Fig. 2. Due to the low rate of ${ }^{9} \mathrm{Li}$ compared to IBD, it is not possible to determine the ${ }^{9} \mathrm{Li}$ rate directly. Instead, an additional neutron capture signal was required to reduce the muon rate. However, the efficiency of this additional requirement for true ${ }^{9} \mathrm{Li}$ is uncertain, and was the dominant uncertainty in the previous measurement at $40 \%$. In this updated analysis with additional statistics, the prompt energy cut is increased to $8 \mathrm{MeV}(6 \mathrm{MeV})$ to $12 \mathrm{MeV}$ in the near halls (far hall). This cut suppresses IBD while preserving $(15 \pm 2) \%$ of ${ }^{9} \mathrm{Li}$ in the near halls and $(40 \pm 3) \%$ in the far hall. These efficiencies are determined from the ${ }^{9} \mathrm{Li}$ prompt energy spectrum, which is measured in data by selecting a ${ }^{9} \mathrm{Li}$-enhanced sample immediately following high-energy muon showers, and subtracting an IBD-pure spectrum from candidates $>1 \mathrm{~s}$ from a muon shower. The uncertainties on

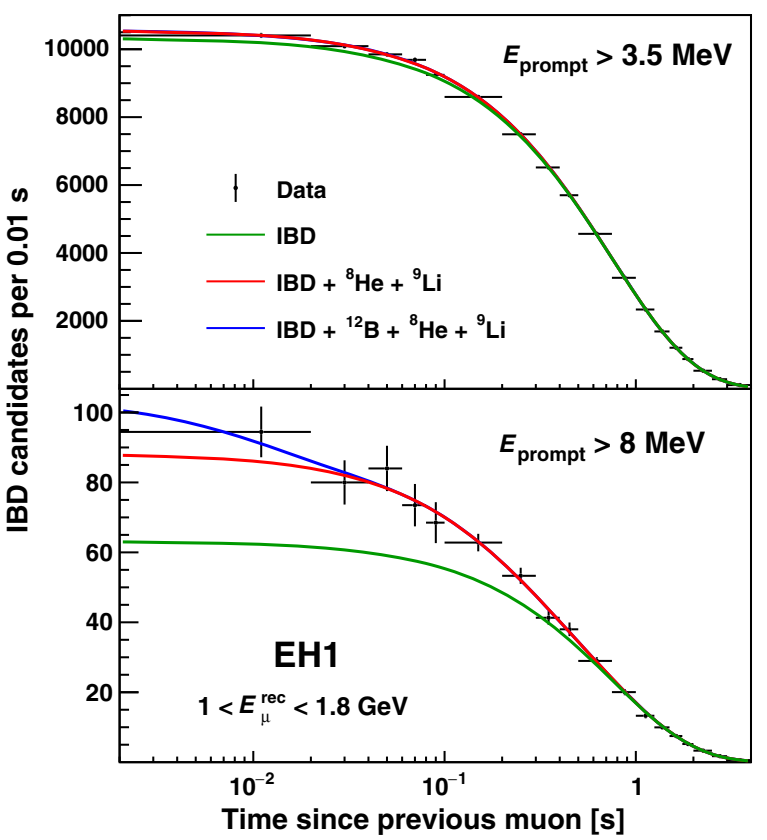

FIG. 2. Time from the IBD candidate to the preceding muon with $1<E_{\mu}^{\mathrm{rec}}<1.8 \mathrm{GeV}$ in EH1. Due to the high muon rate and high IBD rate, the ${ }^{9} \mathrm{Li}$ component (red curve) cannot be accurately measured with a prompt energy cut of $3.5 \mathrm{MeV}$ (top panel). Applying a higher prompt energy cut of $8 \mathrm{MeV}$ reduces the IBD component (green curve), and ${ }^{9} \mathrm{Li}$ yields can be directly determined (bottom panel). The ${ }^{12} \mathrm{~B}$ component is due to a coincidence of two ${ }^{12} \mathrm{~B}$ decays produced in the muon shower (blue curve). 
the prompt cut efficiencies are dominated by the statistics of the ${ }^{9} \mathrm{Li}$-enhanced sample. The bottom panel of Fig. 2 shows the high prompt energy cut applied in EH1. With the higher prompt energy cut, the ${ }^{9} \mathrm{Li}$ rate can be determined without requiring a neutron tag for $E_{\mu}^{\mathrm{rec}}>1 \mathrm{GeV}$.

In the final $\bar{\nu}_{e}$ sample, a veto is applied for $1 \mathrm{~ms}$ following muons with $0.02<E_{\mu}^{\mathrm{rec}}<2.5 \mathrm{GeV}$, and $1 \mathrm{~s}$ for muons with $E_{\mu}^{\text {rec }}>2.5 \mathrm{GeV}$. With these vetoes, $16 \%$ of the ${ }^{9} \mathrm{Li}$ background arises from muons with $E_{\mu}^{\mathrm{rec}}<1 \mathrm{GeV}$, and is estimated using the neutron tag strategy of Ref. [7] with a $(95 \pm 40) \%$ efficiency. The improved ${ }^{9} \mathrm{Li}$ estimate in Table I is consistent with the previous analysis [7], and the uncertainty is reduced from $43 \%$ to $27 \%$ in the near ADs. The updated uncertainty is dominated by the statistics of the ${ }^{9} \mathrm{Li}$-enhanced sample, and can be further reduced by including additional data. The largest systematic uncertainty for $E_{\mu}^{\text {rec }}>1 \mathrm{GeV}$ is $13 \%$, due to the efficiency of the prompt energy cut.

The detection efficiencies are described in detail in Ref. [7]. Correlated uncertainties in efficiency between the near and far sites cancel in the oscillation measurement. The $0.2 \%$ uncertainty in the relative energy scale introduces an uncorrelated $0.08 \%$ uncertainty in the efficiency of the delayed energy cut. The uncertainty in the Gd capture fraction is estimated to be $0.1 \%$ by comparing the capture time distributions of the ADs. Total variation in efficiency among detectors is estimated to be $0.13 \%$, as in Ref. [7]. The measured $\bar{\nu}_{e}$ rates are compared between adjacent detectors in the near experimental halls, in which the statistical uncertainty is of the same order as the uncertainty in the efficiency. The measured ratios are found to be $0.981 \pm 0.002$ between AD1 and AD2 (in the 6 and $8 \mathrm{AD}$ periods) and $1.014 \pm 0.002$ between $\mathrm{AD} 3$ and $\mathrm{AD} 8$ (in the 7 and $8 \mathrm{AD}$ periods). The expected ratios are 0.982 and 1.013 , respectively, accounting for small differences in the baselines and target mass. Comparison in the far hall is also consistent between data and prediction, but dominated by statistical uncertainty. The consistency between measured and predicted ratios provides an independent confirmation of the estimation of the uncorrelated efficiency uncertainty.

The predicted $\bar{\nu}_{e}$ flux includes a $\sim 0.3 \%$ contribution from the spent nuclear fuel present in the cooling pool adjacent to each reactor core [15]. In Ref. [7], the uncertainty of the spent fuel contribution was conservatively set to $100 \%$ due to a lack of knowledge of the spent fuel inventory history. An investigation of the history, in collaboration with the nuclear power plant, results in a reduced uncertainty of $30 \%$, now dominated by the calculation of the $\bar{\nu}_{e}$ spectrum [16], which sums the $\beta$ decay spectra of fission isotopes with half-lives longer than 10 hours.

Oscillation parameters are extracted from the disappearance of $\bar{\nu}_{e}$, as given by the survival probability

$$
\begin{aligned}
P= & 1-\cos ^{4} \theta_{13} \sin ^{2} 2 \theta_{12} \sin ^{2} \Delta_{21} \\
& -\sin ^{2} 2 \theta_{13}\left(\cos ^{2} \theta_{12} \sin ^{2} \Delta_{31}+\sin ^{2} \theta_{12} \sin ^{2} \Delta_{32}\right) \\
\simeq & 1-\cos ^{4} \theta_{13} \sin ^{2} 2 \theta_{12} \sin ^{2} \Delta_{21} \\
& -\sin ^{2} 2 \theta_{13} \sin ^{2} \Delta_{e e},
\end{aligned}
$$

where $\Delta_{i j}=1.267 \Delta m_{i j}^{2} L / E$. Here $E$ is the $\bar{\nu}_{e}$ energy in $\mathrm{MeV}$ and $L$ is the distance in meters from the $\bar{\nu}_{e}$ production point. The oscillation phases due to $\Delta m_{31}^{2}$ and $\Delta m_{32}^{2}$ are degenerate in the range of $L / E$ relevant for this measurement. An effective neutrino mass-squared difference $\Delta m_{e e}^{2}$ is described in the Supplemental Material in Ref. [17]. Solar parameters $\theta_{12}$ and $\Delta m_{21}^{2}$ are fixed to the global average in Ref. [18], and their uncertainties are negligible to this measurement.

The observed background-subtracted signal $N_{i}^{\mathrm{far}, \mathrm{obs}}$ in the $i_{\text {th }}$ energy bin in the far hall is compared to the prediction $N_{i}^{\text {far,pred }}$, given in Eq. (2):

$$
N_{i}^{\mathrm{far}, \text { pred }}=w_{i}\left(\theta_{13}, \Delta m_{\mathrm{ee}}^{2}\right) N_{i}^{\text {near,obs }} .
$$

The predicted rate is based on the measurements in the near halls, $N_{i}^{\text {near,obs }}$, with minimal dependence on models of the reactor $\bar{\nu}_{e}$ flux. Weight factors $w_{i}$ account for the difference in near and far hall measurements, including detection efficiencies, target mass differences, reactor power and distance from each core, and oscillation probability. The 6,8 , and $7 \mathrm{AD}$ periods are treated separately in order to properly handle correlations in reactor $\bar{\nu}_{e}$ flux, detector response, and background.

To evaluate the oscillation parameters, a $\chi^{2}$ is defined in Eq. (3), where the statistical component of the covariance matrix $V$ is estimated analytically, and the systematic component is evaluated from simulations:

$\chi^{2}=\sum_{i, j}\left(N_{j}^{\mathrm{far}, \mathrm{obs}}-N_{j}^{\mathrm{far}, \text { pred }}\right)\left(V^{-1}\right)_{i j}\left(N_{i}^{\mathrm{far}, \mathrm{obs}}-N_{i}^{\mathrm{far}, \text { pred }}\right)$.

This approach is described in detail as Method A in Ref. [7].

Using this method, values of $\sin ^{2} 2 \theta_{13}=0.0856 \pm 0.0029$ and $\Delta m_{e e}^{2}=\left(2.522_{-0.070}^{+0.068}\right) \times 10^{-3} \mathrm{eV}^{2}$ are obtained, with $\chi^{2} / \mathrm{NDF}=148.0 / 154$. Consistent results are obtained using Methods B or C in Ref. [7]. Analysis using the exact $\bar{\nu}_{e}$ disappearance probability for three-flavor oscillations yields $\Delta m_{32}^{2}=\left(2.471_{-0.070}^{+0.068}\right) \times 10^{-3} \mathrm{eV}^{2}\left[\Delta m_{32}^{2}=\right.$ $\left.-\left(2.575_{-0.070}^{+0.068}\right) \times 10^{-3} \mathrm{eV}^{2}\right]$ assuming normal (inverted) hierarchy. Statistics contribute $60 \%(50 \%)$ to the total uncertainty in the $\sin ^{2} 2 \theta_{13}\left(\Delta m_{e e}^{2}\right)$ measurement. The systematic uncertainty of $\sin ^{2} 2 \theta_{13}$ is dominated by the detection efficiency uncertainty uncorrelated among detectors and the reactor $\bar{\nu}_{e}$ flux prediction, while that of $\Delta m_{e e}^{2}$ is dominated by the uncorrelated energy scale uncertainty. 


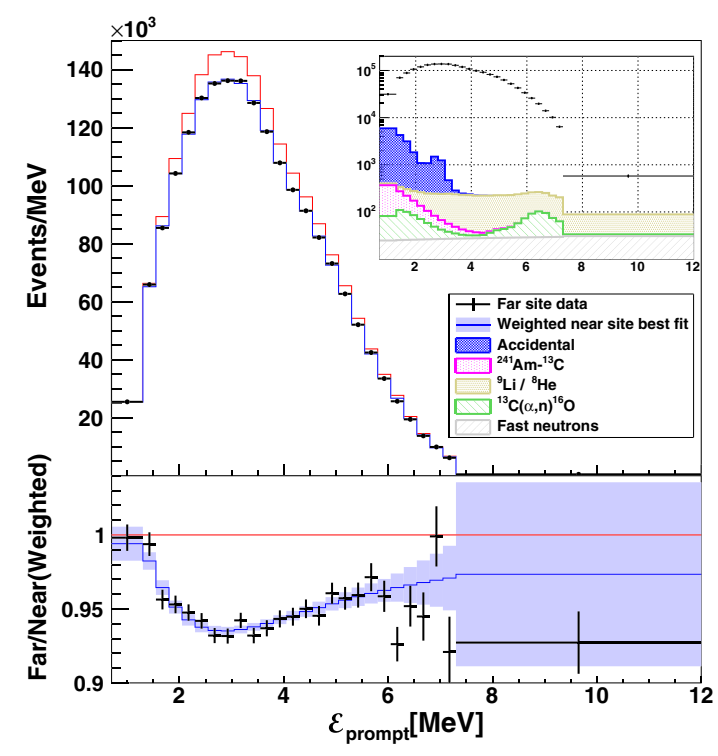

FIG. 3. The background-subtracted spectrum at the far site (black points) and the expectation derived from near-site measurements excluding (red line) or including (blue line) the best-fit oscillation. The bottom panel shows the ratios of data over predictions with no oscillation. The shaded area is the total uncertainty from near-site measurements and the extrapolation model. The error bars represent the statistical uncertainty of the far-site data. The inset shows the background components on a logarithmic scale. Detailed spectra data are provided as Supplemental Material [14].

The reconstructed prompt energy spectrum observed in the far site is shown in Fig. 3, as well as the best-fit predictions. The $68.3 \%, 95.5 \%$, and $99.7 \%$ C.L. allowed regions in the $\Delta m_{e e}^{2}-\sin ^{2} 2 \theta_{13}$ plane are shown in Fig. 4.

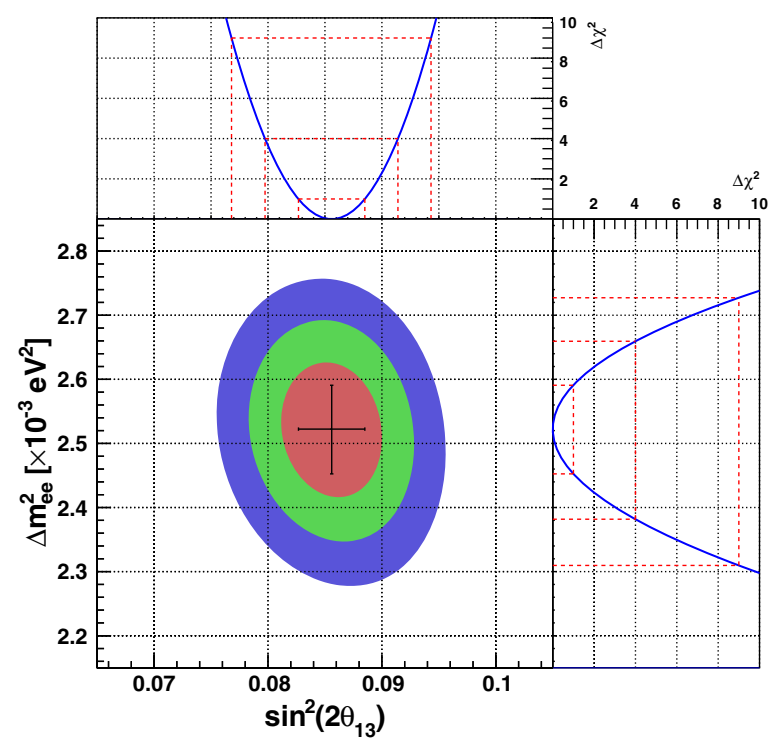

FIG. 4. The $68.3 \%, 95.5 \%$, and $99.7 \%$ C.L. allowed regions in the $\Delta m_{e e^{-}}^{2} \sin ^{2} 2 \theta_{13}$ plane. The one-dimensional $\Delta \chi^{2}$ for $\sin ^{2} 2 \theta_{13}$ and $\Delta m_{e e}^{2}$ are shown in the top and right panels, respectively. The best-fit point and one-dimensional uncertainties are given by the black cross.
In summary, new measurements of $\sin ^{2} 2 \theta_{13}$ and $\Delta m_{e e}^{2}$ are obtained with 1958 days of data and reduced systematic uncertainties. This is the most precise measurement of $\sin ^{2} 2 \theta_{13}$, and the precision of $\Delta m_{32}^{2}$ is comparable to that of the accelerator-based experiments [19-21].

Daya Bay is supported in part by the Ministry of Science and Technology of China, the U.S. Department of Energy, the Chinese Academy of Sciences, the CAS Center for Excellence in Particle Physics, the National Natural Science Foundation of China, the Guangdong provincial government, the Shenzhen municipal government, the China General Nuclear Power Group, Key Laboratory of Particle and Radiation Imaging (Tsinghua University), the Ministry of Education, Key Laboratory of Particle Physics and Particle Irradiation (Shandong University), the Ministry of Education, Shanghai Laboratory for Particle Physics and Cosmology, the Research Grants Council of the Hong Kong Special Administrative Region of China, the University Development Fund of The University of Hong Kong, the MOE program for Research of Excellence at National Taiwan University, National Chiao-Tung University, and NSC fund support from Taiwan, the U.S. National Science Foundation, the Alfred P. Sloan Foundation, the Ministry of Education, Youth, and Sports of the Czech Republic, the Charles University Research Centre UNCE, the Joint Institute of Nuclear Research in Dubna, Russia, the CNFC-RFBR joint research program, the National Commission of Scientific and Technological Research of Chile, and the Tsinghua University Initiative Scientific Research Program. We acknowledge Yellow River Engineering Consulting Co., Ltd., and China Railway 15th Bureau Group Co., Ltd., for building the underground laboratory. We are grateful for the ongoing cooperation from the China General Nuclear Power Group and China Light and Power Company.

*Present address: Department of Chemistry and Chemical Technology, Bronx Community College, Bronx, New York 10453.

[1] F. P. An et al. (Daya Bay Collaboration), Phys. Rev. Lett. 108, 171803 (2012).

[2] J. K. Ahn et al. (RENO Collaboration), Phys. Rev. Lett. 108, 191802 (2012).

[3] Y. Abe et al. (Double Chooz Collaboration), Phys. Rev. Lett. 108, 131801 (2012).

[4] K. Abe et al. (T2K Collaboration), Phys. Rev. Lett. 112, 061802 (2014).

[5] P. Adamson et al. (MINOS Collaboration), Phys. Rev. Lett. 110, 171801 (2013).

[6] F. P. An et al. (Daya Bay Collaboration), Phys. Rev. Lett. 112, 061801 (2014).

[7] F. P. An et al. (Daya Bay Collaboration), Phys. Rev. D 95, 072006 (2017).

[8] F. P. An et al. (Daya Bay Collaboration), Nucl. Instrum. Methods Phys. Res., Sect. A 811, 133 (2016). 
[9] F. P. An et al. (Daya Bay Collaboration), Nucl. Instrum. Methods Phys. Res., Sect. A 773, 8 (2015).

[10] D. Adey et al. (Daya Bay Collaboration), arXiv:1808 .10836 .

[11] Y. Huang et al., Nucl. Instrum. Methods Phys. Res., Sect. A 895, 48 (2018).

[12] P. Huber, Phys. Rev. C 84, 024617 (2011).

[13] T. A. Mueller, D. Lhuillier, M. Fallot, A. Letourneau, S. Cormon, M. Fechner, L. Giot, T. Lasserre, J. Martino, G. Mention, A. Porta, and F. Yermia, Phys. Rev. C 83, 054615 (2011).

[14] See Supplemental Material at http://link.aps.org/ supplemental/10.1103/PhysRevLett.121.241805 for the following tabulated data: the observed prompt energy spectra for $\bar{\nu}_{e}$ inverse $\beta$-decay candidates and estimated backgrounds for each experimental hall, the distribution of
$\Delta \chi^{2}$ versus $\sin ^{2} 2 \theta_{13}$ and $\Delta m_{e e}^{2}$, and the components of the detector response model $P\left(\mathcal{E}_{\text {rec }} ; \mathcal{E}_{\text {true }}\right)$.

[15] F. P. An et al. (Daya Bay Collaboration), Chin. Phys. C 41, 013002 (2017).

[16] X. B. Ma, Y. F. Zhao, Y. X. Chen, W. L. Zhong, and F. P. An, Nucl. Phys. A966, 294 (2017).

[17] F. P. An et al. (Daya Bay Collaboration), Phys. Rev. Lett. 115, 111802 (2015).

[18] M. Tanabashi et al. (Particle Data Group), Phys. Rev. D 98, 030001 (2018).

[19] P. Adamson et al. (MINOS Collaboration), Phys. Rev. Lett. 112, 191801 (2014).

[20] M. A. Acero et al. (NOvA Collaboration), Phys. Rev. D 98, 032012 (2018).

[21] K. Abe et al. (T2K Collaboration), Phys. Rev. Lett. 121, 171802 (2018). 